\title{
Profilaxis antifúngica en niños y adultos sometidos a trasplante de órganos sólidos y de precursores hematopoyéticos
}

\author{
Ricardo Rabagliati y M. Elena Santolaya
}

\section{Prophylaxis against fungal infections in solid organ and hematopoietic stem cells transplantation}

Invasive fungal infections are an important cause of morbidity and mortality in SOT and HSCT recipients. The main species involved are Candida spp. and Aspergillus spp, less frequently Cryptococcus spp., causal agents of mucormycosis and Fusarium spp. Usually occur within the first six months post-transplant, but they do it later, especially during episodes of rejection, which maintains the state of immune system involvement. Prophylaxis recommendations are specific to each type of transplant. In liver transplantation use of fluconazole is recommended only in selected cases by high risk factor for invasive fungal infections (A1). If the patient has a high risk of aspergillosis, there are some suggestions for adults population to use amphotericin B-deoxycholate, liposomal amphotericin B or caspofungin (C2) without being validated none of these recommendations in pediatric population. In adult lung transplant patients where the risk of aspergillosis is higher than in other locations, we recommend universal prophylaxis with itraconazole $200 \mathrm{mg} /$ day, nebulised liposomal amphotericin B or voriconazole (C2), no validated recommendations for pediatrics. In HSCT, universal prophylaxis is recommended only in allogeneic and autologous selected cases. The most accepted indication is fluconazole (A1), and posaconazole (A1) or micafungin (A1) in selected cases with high risk of aspergillosis.

Key words: Fungus, Candida, Aspergillus, yeasts, moulds, prophylaxis, amphotericin, fluconazole, voriconazole, posaconazole, caspofungin, micafungin, transplants, solid organ transplantation, hematopoietic stem cells transplantation.

Palabras clave: Hongos, Candida, Aspergillus, levaduras, hongos filamentosos, profilaxis, anfotericina, fluconazol, voriconazol, posaconazol, caspofungina, micafungina, trasplante, trasplante de órganos sólidos, trasplante de precursores hematopoyéticos.

\section{Introducción}

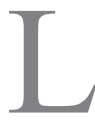

as infecciones fúngicas constituyen una importante causa de morbilidad en los pacientes receptores de trasplantes de órganos sólidos (TOS) y trasplante de precursores hematopoyéticos $(\mathrm{TPH})^{1}$, generando desafíos diagnósticos y terapéuticos, elevación de los costos de manejo y elevada mortalidad ${ }^{2,3}$. En los receptores de trasplantes, la enfermedad fúngica invasora (EFI) es provocada tanto por hongos levaduriformes como filamentosos, destacando Candida spp. y Aspergillus spp..$^{2-4}$, menos frecuentemente Cryptococcus spp., agentes causales de mucormicosis ${ }^{5,6}$ y Fusarium spp. ${ }^{7}$, infecciones por hongos endémicos como Histoplasma capsulatum, Coccidioides immitis, Paracoccidioides brasiliensis, y con menor cuantía dematiáceos como Cladosporium, Cladophialophora, Scopulariopsis, Alternaria, Curvularia y Bipolaris $\mathrm{spp}^{8}$.

Existen diferencias en la frecuencia de EFI entre los distintos tipos de TOS y TPH; no obstante, algunas características son comunes: en ambos grupos la mayoría de las EFI son producidas por Candida spp. o Aspergillus spp., ocurren dentro de los primeros seis meses post-trasplante y pueden presentarse como enfermedad localizada o diseminada, comprometiendo dos o más órganos. Cada vez con mayor frecuencia, las EFI se presentan en forma más tardía, especialmente durante episodios de rechazo, en que se mantiene el estado de compromiso del sistema inmune.

En general, las infecciones por Candida spp. son originadas desde una fuente endógena, mientras que las producidas por hongos filamentosos se originan desde fuentes exógenas medio-ambientales ${ }^{2}$. Durante años la mortalidad descrita, tanto en niños como en adultos, ha sido muy elevada, alcanzando a $60 \%$ o más en casos de aspergilosis en TPH y TOS 9 . Hoy en día, las nuevas estrategias diagnósticas y el uso de nuevos antifúngicos han mejorado el pronóstico de estos pacientes, describiéndose una mortalidad reciente en candidiasis de 10 a $35 \%$ y en aspergilosis de 35 a 50\% en niños y adultos sometidos a $\mathrm{TPH}^{10-12}$. En este escenario, se requiere un enfrentamiento de prevención, diagnóstico y tratamiento racional de las EFI con la intención de aminorar su impacto en la morbilidad y mortalidad.

A fin de establecer las recomendaciones de profilaxis
Departamento de Enfermedades Infecciosas, Escuela de Medicina Pontificia Universidad Católica de Chile, Santiago (RB). Unidad de Infectología Hospital Dr. Luis Calvo Mackenna, Santiago. Facultad de Medicina, Universidad de Chile (MES).

Los autores declaran no tener conflictos de interés.

Correspondencia a: rabagliati@med.puc.cl msantola@med.uchile.cl 
antifúngica se presentará la epidemiología en cada grupo así como los factores de riesgo. Las medidas de prevención de EFI incluyen recomendaciones de intervención en el ambiente y medidas de profilaxis farmacológica, con diferentes énfasis según la temporalidad post-trasplante, los factores de riesgo y la epidemiología institucional.

\section{Epidemiología}

\section{Trasplantes de órganos sólidos}

En la literatura científica internacional se describen frecuencias de $60 \%$ de EFI en receptores de trasplante intestinal, $40 \%$ en receptores de trasplante pulmonar, hepático y pancreático y $14 \%$ en receptores de trasplante renal. Estas cifras reflejan la magnitud del problema en series publicadas mayoritariamente durante la década de los $90^{13-16}$. Recientemente Pappas y cols., publicaron resultados de un registro prospectivo de EFI en 15 centros de trasplante en E.U.A., entre los años 2001 y 2006. En una cohorte de 16.808 receptores de TOS, hubo $1.208 \mathrm{EFI}$ en 1.063 pacientes, predominantemente adultos, con una incidencia anual de $1,95 \%$ para candidiasis y $0,65 \%$ para aspergilosis. Del conjunto de EFI, candidiasis resultó ser la más frecuente $(53 \%)$, seguida de aspergilosis (19\%), criptococosis ( $8 \%$ ), hongos filamentosos no-Aspergillus $(8 \%)$, micosis endémicas $(5 \%)$ y mucormicosis $(2 \%)$. De acuerdo al tipo de trasplante, la incidencia acumulada anual fue más elevada para intestino $(11,6 \%)$, seguida de pulmón $(8,6 \%)$, hígado $(4,7 \%)$, corazón $(4 \%)$, páncreas $(3,4 \%)$ y la menor incidencia fue en receptores de trasplante de riñón $(1,3 \%)^{17}$.

Entre los receptores de hígado, intestino, páncreas y riñón, la candidiasis resulta ser la EFI más frecuente ${ }^{17}$. Sin embargo, la aspergilosis es significativamente más frecuente en receptores de trasplante pulmonar, $(12,8 \mathrm{vs}$ $0,4 \%$ observada en otros tipos de TOS $)^{18}$. También en receptores de trasplante cardíaco, la aspergilosis es la infección predominante describiéndose hasta en 14\% ${ }^{19-22}$. Un estudio multicéntrico italiano, que incluyó la visión retrospectiva de 1.852 trasplantes cardíacos, describe que $2,2 \%$ presentó una EFI, siendo aspergilosis la más frecuente $(64 \%)$, seguido de candidiasis $(23 \%)$. La mortalidad descrita en esta serie para receptores de trasplante de órganos torácicos con candidiasis y aspergilosis es de 33 y $29 \%$, respectivamente ${ }^{23}$.

Criptococosis se presenta con mayor frecuencia en receptores de trasplante cardíaco e intestinal que en pacientes con trasplante renal o pulmonar ${ }^{24}$, con una mortalidad de $21 \%{ }^{25}$.

La frecuencia y el tipo de EFI varían además de acuerdo a la temporalidad post-trasplante ${ }^{2}$. Durante el primer período (0-30 días), suelen observarse infecciones fúngicas relacionadas a la cirugía, procedimientos invasores y cuidados hospitalarios en unidades de paciente crítico. En esta etapa, predominan las infecciones por Candida spp., con variaciones de frecuencia de $C$. albicans vs noalbicans, según la epidemiología de cada institución. Durante el segundo período (31 a 180 días) el efecto neto de la inmunosupresión tiene su máxima expresión por lo que predominan las infecciones por Aspergillus spp., seguido por otros hongos filamentosos, reactivaciones de micosis endémicas o criptococosis. En el tercer período, cumplidos los seis meses, las infecciones fúngicas son infrecuentes y suelen asociarse a períodos de inmunosupresión en el contexto de elevación de dosis de inmunosupresores por rechazo o infecciones por citomegalovirus $(\mathrm{CMV})^{26}$. Sin embargo, de acuerdo a datos recientes, se describe un desplazamiento de las EFI en TOS, con medianas de tiempo para manifestación de candidiasis, aspergilosis y criptococosis de 103, 184 y 575 días, respectivamente ${ }^{17}$. Estos cambios pueden relacionarse a la duración de la profilaxis, la necesidad de aumentar la inmunosupresión o exposiciones medioambientales tales como las que se pueden presentar en eventos climáticos o en construcciones que se han asociado a brotes de aspergilosis.

En suma, la EFI es un problema que afecta más frecuentemente a pacientes sometidos a trasplante de intestino, páncreas, hígado, pulmón y corazón, siendo candidiasis la EFI más frecuente en los trasplantes no torácicos y aspergilosis en trasplantes de pulmón y corazón. Es importante recalcar que las EFIs no constituyen un problema relevante en los receptores de trasplante renal. Si bien no contamos con datos nacionales publicados de EFI en TOS, es planteable que el predominio de un tipo de agente sobre otro en los distintos grupos de órganos se presente de una manera similar.

\section{Trasplante de precursores hematopoyéticos}

La frecuencia de EFI en este grupo puede ser tan alta como 25\% según datos de autopsias. Los hongos más frecuentemente identificados son Candida spp. y Aspergillus spp., con menor frecuencia siguen el Orden Mucorales (agentes de mucormicosis), Fusarium, Scedosporium spp. y otros hongos emergentes ${ }^{27}$.

Datos recientes de pacientes receptores de $\mathrm{TPH}$, reportan incidencias de EFI de 3,7\% ${ }^{28}$; sin embargo, al comparar trasplante autólogo vs alogénico, las magnitudes son significativamente menores en autólogo: $1,2^{28}$ vs $7,8-8 \%$ en alogénico ${ }^{28,29}$. Según el tipo de EFI específica, se describen frecuencias de $3 \%$ para candidiasis y $8 \%$ para aspergilosis en TPH, que se elevan hasta $10,5 \%$ si se considera sólo TPH alogénico ${ }^{29-31}$. Datos del estudio español RESISTRA, que evaluó $1.318 \mathrm{TPH}$ de forma prospectiva en dos años, determinó una frecuencia de $6,7 \%$ de EFI, con predominio de $C$. no-albicans, seguido de aspergilosis, con diferencias significativas en TPH autólogo $(1,2 \%)$ vs alogénico $(8,3 \%)^{32}$. 
En relación a la temporalidad post-TPH y presentación de una EFI, durante el primer período post-trasplante (0 a 30 días), que se extiende desde la infusión hasta después del implante, se presenta habitualmente a las 2 a 3 semanas; las infecciones están asociadas a la profundidad y duración de la neutropenia y a la intensidad de la mucositis, por lo que en esta etapa prevalecen Candida spp. y Aspergillus spp. En la segunda fase (día 30 al 100), los principales factores de riesgo son el uso de inmunosupresores, principalmente corticosteroides, el desarrollo de una enfermedad de injerto contra hospedero (EICH) y la infección por CMV, predominando en este período Aspergillus spp. y mucorales. En la tercera fase, después del día 100, el riesgo de infección depende de la presencia de una EICH crónica y la intensidad de su tratamiento, lo que en definitiva se relaciona con el estado de inmuno-reconstitución. En este período, Aspergillus spp. sigue siendo el agente prevalente. En el estudio español RESISTRA, 41\% de los casos de aspergilosis se presentaron en la fase neutropénica, $46 \%$ en la postimplante hasta el día 180 y $12 \%$ después de los seis meses de efectuado el trasplante ${ }^{32}$.

\section{Factores de riesgo}

Son factores de riesgo universales para el desarrollo de una EFI la neutropenia grave y prolongada, la inmunodeficiencia celular, la presencia de mucositis, el antecedente de procedimientos invasores, la infección por $\mathrm{CMV}$, el uso de antimicrobianos de amplio espectro y el uso de corticoesteroides $^{11,33}$.

\section{Trasplante de órganos sólidos}

En el grupo de pacientes receptores de trasplante hepático, se han descrito como factores de riesgo específicos para candidiasis las transfusiones intra o peri-operatorias, en magnitud superior a 10 unidades de eritrocitos, estadía en UCI $>5$ días post-trasplante, creatininemia basal preoperatoria $>2,0 \mathrm{mg} / \mathrm{dL}$ o necesidad de diálisis, anastomosis bilio-digestiva, trombosis de la arteria hepática, re-trasplante, falla hepática fulminante pre-trasplante, rechazo grave, colonización por Candida en los primeros tres días post-trasplante e infección por $\mathrm{CMV}^{34}$. Para infección por Aspergillus spp., se han identificado como factores de riesgo específicos el re-trasplante, la infección por CMV y la necesidad de diálisis post-trasplante ${ }^{35}$.

En niños que reciben un trasplante hepático se han identificado otras variables asociadas al desarrollo de EFI, como son el uso de tacrolimus, la colonización fúngica pre-trasplante, la infección bacteriana y por virus de Epstein Barr en el post-trasplante ${ }^{36}$.

Específicamente, en un estudio que incluyó 50 casos de mucormicosis, se identificó que en el grupo de receptores de trasplante hepático era particularmente frecuente la infección diseminada, y se logró determinar como factores de riesgo la presencia de insuficiencia renal, diabetes mellitus y uso previo de voriconazol o caspofungina ${ }^{37}$.

En trasplante cardíaco, aspergilosis es la EFI más frecuente. Entre los factores de riesgo identificados se encuentran la re-intervención quirúrgica, enfermedad por $\mathrm{CMV}$, insuficiencia renal y la existencia de casos de aspergilosis en los dos meses previos en la unidad de hospitalización del paciente ${ }^{38}$. En trasplante pulmonar se han descrito como factores de riesgo para aspergilosis la colonización de la vía aérea por Aspergillus spp., la infección por $\mathrm{CMV}$, la hipogamaglobulinemia ${ }^{18}$ y se han comunicado brotes de aspergilosis en este grupo de pacientes expuestos a construcciones en el hospital ${ }^{39}$.

\section{Trasplante de precursores hematopoyéticos}

En TPH, la frecuencia y tipo de EFI dependen del momento del trasplante. Infecciones por Candida spp. y Aspergillus spp. son las más frecuentes durante el primer período post TPH y Aspergillus spp. es la etiología más frecuente en las etapas siguientes; mucho menos frecuente son las mucormicosis y fusariosis ${ }^{40}$. Los factores de riesgo identificados para infección por Candida spp. en el primer período post-TPH son la profundidad de la neutropenia, la presencia de mucositis y el uso de CVC, agregándose en el segundo período post-trasplante la presencia de EICH intestinal $^{41}$.

El principal factor de riesgo para adquirir una aspergilosis durante el primer período post TPH es la neutropenia prolongada; por lo tanto, son de mayor riesgo los pacientes que presentan neutropenia previa al trasplante y los receptores de trasplantes de cordón umbilical y trasplante haploidéntico, mientras que son de menor riesgo los pacientes que reciben trasplante de precursores de sangre periférica o aquellos en que el acondicionamiento no es mieloablativo ${ }^{40}$. Otro factor importante es la exposición ambiental a esporas fúngicas que pueden estar presentes en el aire o en el agua ${ }^{42}$.

En las fases siguientes al implante medular, el principal factor de riesgo para contraer una aspergilosis es la inmunosupresión producto de la EICH y su tratamiento, particularmente el uso de corticoesteroides ${ }^{40,43}$, además de la infección/enfermedad por $\mathrm{CMV}^{31}$. Otros factores de riesgo, menos explorados, son algunos aspectos geoclimáticos, como la presencia de estaciones cálidas y húmedas ${ }^{44} \mathrm{y}$ factores del hospedero, como el aumento de los depósitos de hierro ${ }^{45}$ y la presencia de polimorfismos de receptores $\mathrm{TL}^{46}$.

\section{Antifúngicos profilácticos}

Las estrategias de profilaxis deben incluir medidas generales de disminución de la exposición a los micro- 
organismos causantes y medidas de control de los riesgos propios del paciente de acuerdo a su trasplante, ya descritos en párrafos anteriores. Con el objetivo de disminuir la exposición a Candida de una fuente exógena, se debe exigir el cumplimiento de la adecuada higiene de manos del personal de salud. A fin de disminuir la exposición a esporas de Aspergillus spp., los hospitales que realizan trasplantes deben contar con un manejo adecuado del aire mediante la incorporación de filtros HEPA que permitan disponer espacios con ambiente protegido para los pacientes de mayor riesgo y una política estricta de control de infecciones en relación a construcciones.

Se presenta a continuación la evidencia disponible, según el tipo de trasplante, para el uso de antifúngicos profilácticos.

\section{Trasplante de órganos sólidos}

Trasplante hepático. La efectividad de la profilaxis ha sido evaluada en distintos estudios, buscando evidenciar cuál es la mejor estrategia, usar antifúngicos en forma universal o sólo en aquellos pacientes con factores de riesgo para desarrollar una $\mathrm{EFI}^{47}$.

En un estudio publicado a fines de los 90, Winston y cols. ${ }^{48}$, demostraron que fluconazol comparado con placebo reducía de manera significativa las infecciones invasoras (6 vs 23\%). Estos resultados han sido posteriormente confirmados en una revisión sistemática de la Cochrane que incluyó nueve estudios con pacientes randomizados a recibir profilaxis con un antifúngico vs placebo, demostrando utilidad de fluconazol en reducir las EFIs probadas (RR 0,28. I.C. 95\% 0,13-0,57), sin demostrar disminución de mortalidad (RR 0,90. I.C. 95\% $0,57-1,44)$. Según la misma fuente, para prevenir un caso de EFI se requeriría tratar a seis pacientes con fluconazol ${ }^{49}$. En esta revisión no se evidenció que el uso de fluconazol incrementara significativamente infecciones por especies resistentes a este fármaco, lo que contrasta con un metaanálisis publicado posteriormente que demuestra mayor frecuencia de infecciones por C. no-albicans, en particular C. glabrata en los pacientes que recibieron fluconazol profiláctico $^{50}$.

Considerando estos datos, existe evidencia suficiente para recomendar uso de profilaxis con fluconazol sólo en pacientes que presentan al menos uno de los factores de riesgo descritos ${ }^{51}$ (transfusiones intra o peri-operatorias superiores a 10 unidades de eritrocitos, estadía en UCI $>5$ días post-trasplante, creatininemia basal pre-operatoria $>2,0 \mathrm{mg} / \mathrm{dL}$ o necesidad de diálisis, anastomosis biliodigestiva, trombosis de la arteria hepática, re-trasplante, falla hepática fulminante pre-trasplante, rechazo grave, colonización por Candida en los primeros tres días post-trasplante e infección por CMV), en dosis de 400 $\mathrm{mg}$ /día intravenoso (iv) u oral (vo) por cuatro semanas, corrigiendo la dosis según fuese la función renal.
En el grupo de pacientes en riesgo de infección por Aspergillus spp. se puede considerar una profilaxis alternativa con bajas dosis de anfotericina B, $15 \mathrm{mg} /$ día iv o anfotericina liposomal, $50 \mathrm{mg} /$ día, aunque la dosis y duración efectiva no han sido establecidas ${ }^{52}$. En un estudio de Singh y cols., que incluyó una cohorte histórica de pacientes sin profilaxis más una cohorte en la que sí se usó profilaxis con anfotericina liposomal en pacientes con alto riesgo de infección fúngica, se observó una significativa disminución de la frecuencia de EFI ${ }^{53}$. El uso de anfotericina liposomal vía nebulización no ha sido explorado adecuadamente en este grupo de pacientes sometidos a trasplantes.

Otra alternativa evaluada ha sido el uso de caspofungina por al menos 21 días, en un estudio no comparativo que incluyó 71 adultos receptores de trasplante hepático con alto riesgo de adquirir una EFI. La eficacia fue definida como ausencia de emergencia de EFI en los 100 días desde el inicio de la profilaxis. En esta serie se observaron dos casos de infección fúngica, una candidiasis y una mucormucosis, ambos en el sitio de la herida quirúrgica ${ }^{54}$.

Recomendación No 1 (a):
Profilaxis antifúngica en trasplante hepático
Uso sólo en casos seleccionados por factores de riesgo. Alternativas:
- Fluconazol (A1)
- Adultos: $400 \mathrm{mg} /$ día
- Niños: $6-8 \mathrm{mg} / \mathrm{kg} /$ día
- Si existe riesgo de aspergilosis
- Anfotericina B $15 \mathrm{mg} /$ día iv (C2)
- Anfotericina liposomal 50 mg/día iv (C2)
- Caspofungina $70 \mathrm{mg}$ carga inicial luego 50 mg/día iv (C2)
- Ninguno de los tres está validado en pediatría como profilaxis

Trasplante pulmonar. Existen pocos estudios sobre profilaxis en este grupo de pacientes. Husain y cols..$^{55}$, publicaron un estudio comparativo en adultos receptores de trasplante pulmonar, que analizó voriconazol vo vs itraconazol vo, con o sin anfotericina nebulizada, al momento del primer cultivo positivo, evidenciando una menor frecuencia de aspergilosis invasora (1,5 vs 23\%). Este estudio mostró una alta frecuencia de toxicidad hepática en los pacientes que usaron voriconazol. A pesar de la falta de mayores estudios, algunos grupos recomiendan el uso de anfotericina liposomal en nebulización a fin de disminuir la frecuencia de aspergilosis en estos pacientes ${ }^{56}$. Su uso podría complementarse con itraconazol, de acuerdo a los datos del grupo de Cleveland, quienes lograron disminuir la incidencia de aspergilosis a través de la práctica de profilaxis universal con uso de anfotericina nebulizada durante las primeras semanas, seguida de itraconazol $200 \mathrm{mg} /$ día vía oral de forma permanente, reduciendo la frecuencia de 18,2 a $4,9 \%{ }^{18}$. 
Recomendación No 1 (b):

Profilaxis antifúngica en trasplante pulmonar

Uso universal. Alternativas:

- Itraconazol 200 mg/día vo (suspensión), con o sin anfotericina liposomal nebulizada en la fase inicial (C2)

- Anfotericina liposomal nebulizada (C3)

- Voriconazol $200 \mathrm{mg} \mathrm{c/} 12 \mathrm{~h}$ vo (C2)

- No hay recomendación específica en pediatría

Trasplante cardiaco. Existen pocos estudios que hayan evaluado el uso de profilaxis antifúngica. Muñoz y cols., analizaron el uso de itraconazol $400 \mathrm{mg} /$ día en cápsulas en adultos sometidos a trasplante cardíaco. $\mathrm{Su}$ estudio muestra menor frecuencia de aspergilosis, medida como mayor tiempo libre de infección (RR 0,2 I.C. 95\% $0,04-0,9 \mathrm{p}=0,03$ ) y mayor sobrevida al año en aquellos sujetos que recibieron profilaxis (RR 0,5 I.C. 95\% 0,3 $0,8 \mathrm{p}=0,01)$. Los autores recomiendan uso de profilaxis al menos en pacientes con condiciones predisponentes, tales como re-intervención quirúrgica, infección por $\mathrm{CMV}$, necesidad de diálisis post-trasplante y presencia de aspergilosis en otros pacientes de la misma unidad clínica en los últimos dos meses ${ }^{38}$.

Recomendación $N^{\circ} 1$ (c):

Profilaxis antifúngica en trasplante cardíaco

Uso en casos seleccionados por factores de riesgo:

- Itraconazol 200 - 400 mg/día vo (suspensión) (C2)

- No hay recomendación específica en pediatría

Trasplante de precursores hematopoyéticos. De acuerdo a un estudio randomizado, doble ciego, placebocontrolado, publicado por Goodman y cols., a inicios de los años 90, que demostró una reducción significativa en la frecuencia de candidiasis sistémica de 15,8 a 2,8\%, sin diferencias en mortalidad, en adultos sometidos a TPH, se estableció que en este tipo de trasplante el antifúngico de elección era fluconazol $400 \mathrm{mg} /$ día iv o vo, por plazo mínimo de cuatro semanas, corrigiendo la dosis según la función renal, e iniciado durante el acondicionamiento ${ }^{57}$. Un estudio ulterior que prolongó el seguimiento de los pacientes permitió evidenciar que entre los que prolongaban la profilaxis hasta el día 75 post-trasplante, se observaba menor frecuencia de candidiasis, menor muerte relacionada a candidiasis y mayor sobrevida global ${ }^{58}$.

A partir del estudio de Goodman ${ }^{57}$ se estableció en las guías clínicas de los centros de trasplante el uso de fluconazol; sin embargo, posteriormente se ha observado que su uso se relaciona con un cambio epidemiológico produciéndose un predominio de Candida no-albicans ( $C$. glabrata, C. krusei, C. parapsilosis) sobre C. albicans ${ }^{59}$.
En relación a la alternativa de utilizar itraconazol oral como profilaxis, un estudio randomizado, doble-ciego, placebo-controlado que incluyó pacientes adultos con cáncer hematológico o TPH, comparó itraconazol en suspensión vs placebo, determinando una menor frecuencia de infección fúngica superficial/sistémica en el grupo que recibió itraconazol ( 6 vs $15 \% ; \mathrm{p}=0,03)$. Específicamente en el grupo con neutropenia profunda y prolongada se diagnosticó menor número de casos de EFI (6 vs 19\%; $\mathrm{p}=0,04)$ y hubo menor uso de anfotericina-B empírica ${ }^{60}$. El mayor problema en esta serie fue la mala tolerancia de itraconazol reportada por los pacientes. Este inconveniente ha sido observado también en otros estudios que han evidenciado menor frecuencia de infecciones fúngicas con itraconazol comparado con fluconazol ( 9 vs 25\%; $\mathrm{p}=0,01$ ), pero una significativa mayor frecuencia de efectos adversos gastrointestinales (24 vs 9\%; $\mathrm{p}=0,02)^{61}$; o necesidad de suspender la terapia con itraconazol por toxicidad, comparado con el uso de fluconazol (36 vs 16\%) $(\mathrm{p}<0,01)^{62}$.

Es importante recalcar que en Chile no existe la presentación de itraconazol en solución oral y que la presentación en cápsulas no se considera una buena alternativa, debido a su limitada biodisponibilidad.

Buscando una mejor alternativa para prevenir la aparición de aspergilosis, sin los efectos adversos de itraconazol, se han evaluado los nuevos azoles. Un estudio retrospectivo observacional evaluó la incidencia de aspergilosis invasora en adultos receptores de TPH que recibieron voriconazol $v s$ otras terapias, observando ausencia de casos entre 92 pacientes que recibieron voriconazol y una frecuencia de $10 \%(23 / 223)$ entre aquellos que recibieron algún otro antifúngico sistémico profiláctico $(\mathrm{p}<0,05)^{63}$.

Más adelante, posaconazol fue comparado con fluconazol en pacientes adultos receptores de TPH con EICH, observándose menor frecuencia de infecciones invasoras en el grupo que utilizó posaconazol vs fluconazol (7 vs $14 \% ; \mathrm{p}<0,05)$ y específicamente menor frecuencia de aspergilosis $(2 v s 7 \% ; \mathrm{p}<0,05)^{64}$.

En los estudios que han comparado anfotericina deoxicolato con fluconazol no se ha logrado demostrar superioridad de anfotericina pero sí una mayor frecuencia de efectos adversos (19 vs 0\% $)^{65}$, haciendo no recomendable la indicación de anfotericina deoxicolato para estos efectos. Anfotericina liposomal, en dosis de $50 \mathrm{mg}$ iv cada $48 \mathrm{~h}$ ha sido evaluada $v$ s placebo en 132 pacientes adultos con cáncer hematológico, demostrando disminución de EFIs y de la mortalidad atribuible ${ }^{66}$. No hay, sin embargo, evaluaciones de anfotericina liposomal como profilaxis en pacientes receptores de TPH. Tampoco se ha evaluado en este grupo el uso de anfotericina liposomal en inhalación, sólo existen datos en un estudio vs placebo, controlado, en pacientes neutropénicos no sometidos a trasplantes, en que se evidenció disminución de la incidencia de aspergilosis pulmonar ${ }^{67}$. 
De las equinocandinas, micafungina ha sido evaluada como profilaxis, en un estudio randomizado, doble ciego, multicéntrico, que incluyó tanto TPH autólogo (46\%) como alogénico, en fase neutropénica, con una rama de micafungina en 425 pacientes vs 457 con fluconazol, con menor frecuencia de aspergilosis $(0,2$ vs $1,5 \%)$, y de candidemia $(0,4$ vs $0,9 \%)$, demostrando mayor eficacia global ( 80 vs 74\%). Sin embargo, este estudio incluye casos catalogados como infección fúngica posible, por lo que no es comparable con resultados de otros estudios que incluyen sólo casos probables y probados ${ }^{68}$.

La mayoría de los TPH autólogos tiene bajo riesgo de complicarse con una EFI, por lo que no se recomienda profilaxis de rutina en este tipo de trasplante. Sí se recomienda en pacientes adultos que han tenido linfoma, leucemia o mieloma, si se estima que presentarán neutropenia prolongada o mucositis por un acondicionamiento intensivo y en aquellos que han recibido fludarabina o 2-CDA (cladribine, 2-cloro-deoxi-adenosina) en los seis meses previos ${ }^{40}$.

En resumen, los azoles han sido ampliamente estudiados y mayormente validados sobre otras familias de antifúngicos. De acuerdo a la evidencia disponible, se ha planteado iniciar profilaxis con fluconazol hasta completar 75 días, y sólo considerar el uso de posaconazol en casos muy seleccionados ${ }^{69}$. La recomendación de ambos antifúngicos se sustenta en estudios bien diseñados que muestran resultados categóricos de reducción de incidencia de infecciones fúngicas; sin embargo, cabe comentar el riesgo de emergencia de otras infecciones en la medida que se van incorporando distintos antifúngicos. Así como el mayor uso de fluconazol se ha asociado a la mayor frecuencia de Candida no-albicans, el mayor uso de nuevos azoles y equinocandinas también puede determinar cambios epidemiológicos, por lo que la decisión de incorporar cada profilaxis debe ser cuidadosa y asociada a un programa de vigilancia que permita detectar oportunamente estos cambios.

\section{Resumen}

Las infecciones fúngicas invasoras constituyen una importante causa de morbilidad y mortalidad en los pacientes receptores de TOS y TPH. Los principales
Recomendación № 1 (d): Profilaxis antifúngica en

trasplante de precursores hematopoyéticos

Uso universal en trasplante alogénico y, en casos seleccionados, en trasplantes autólogos. Alternativas:

- Fluconazol (A1)

Adultos: 400 mg/día por 30 días (A1), prolongar por 75 días (B1)

Niños: 6-8 mg/kg/día por 30 días, (extrapolado de la experiencia en adultos)

- Posaconazol 200 mg 3 veces al día vo en caso de EICH III-IV (A1)

- Micafungina $50 \mathrm{mg} / \mathrm{d}$ iv durante la fase de neutropenia (A1)

- No se recomienda emplear itraconazol por su mala tolerancia gastrointestinal (D1)

- No se recomienda emplear anfotericina B por toxicidad (D1)

- Ninguno validado con evidencia A1 en pediatría como profilaxis

agentes involucrados son Candida spp. y Aspergillus spp, menos frecuentemente Cryptococcus spp., agentes causales de mucormicosis y Fusarium spp. Se presentan habitualmente dentro de los primeros seis meses posttrasplante, pero también lo hacen en forma más tardía, especialmente durante episodios de rechazo, en que se mantiene el estado de compromiso del sistema inmune. Existen recomendaciones de profilaxis específicas para cada tipo de trasplante. En trasplante hepático se recomienda el uso de fluconazol sólo en casos seleccionados por factores de riesgo (A1). Si existe riesgo de aspergilosis, hay algunas sugerencias en adultos para el uso de anfotericina B-deoxicolato, anfotericina liposomal o caspofungina (todo en categoría $\mathrm{C} 2$ ), sin estar validada ninguna de estas recomendaciones en pediatría. En trasplante pulmonar en paciente adulto, donde el riesgo de aspergilosis es superior a otras localizaciones, se recomienda profilaxis universal, con itraconazol 200 $\mathrm{mg}$ /día, anfotericina liposomal nebulizada o voriconazol (C2), sin recomendaciones validadas para pediatría. En $\mathrm{TPH}$, se recomienda profilaxis universal en trasplante alogénico y sólo para casos seleccionados en trasplantes autólogos. La indicación más aceptada es fluconazol (A1), siendo alternativas a evaluar dependiendo del riesgo de aspergilosis, posaconazol (A1) y micafungina (A1).

\section{Referencias bibliográficas}

1.- Alexiou K, Matschke K, Westphal A, Stangl K, Dschietzig T. Relaxin is a candidate drug for lung preservation: relaxin-induced protection of rat lungs from ischemia-reperfusion injury. J Heart Lung Transplant 2010; 29: 454-60.

2.- Patterson J E. Epidemiology of fungal infections in solid organ transplant patients. Transpl Infect
Dis 1999; $1: 229-36$.

3.- Martino R, Subira M, Rovira M, Solano C, Vázquez L, Sanz G F, et al. Invasive fungal infections after allogeneic peripheral blood stem cell transplantation: incidence and risk factors in 395 patients. Br J Haematol 2002; 116: 475-82.

4.- Morgan J, Wannemuehler K A, Marr K A, Hadley S, Kontoyiannis D P, Walsh T J, et al.
Incidence of invasive aspergillosis following hematopoietic stem cell and solid organ transplantation: interim results of a prospective multicenter surveillance program. Med Mycol 2005; 43 Suppl 1: S49-58.

5.- Upton A, Marr K A. Emergence of opportunistic mould infections in the hematopoietic stem cell transplant patient. Curr Infect Dis Rep 2006; 8: 434-41. 
6.- Brewin J, Mancao C, Straathof K, Karlsson H, Samarasinghe S, Amrolia P J, et al. Generation of EBV-specific cytotoxic T cells that are resistant to calcineurin inhibitors for the treatment of posttransplantation lymphoproliferative disease. Blood 2009; 114: 4792-803.

7.- Nucci M, Marr K A, Queiroz-Telles F, Martins C A, Trabasso P, Costa S, et al. Fusarium infection in hematopoietic stem cell transplant recipients. Clin Infect Dis 2004; 38: 1237-42.

8.- $\quad$ Singh N, Chang F Y, Gayowski T, Marino I R. Infections due to dematiaceous fungi in organ transplant recipients: case report and review. Clin Infect Dis 1997; 24: 369-74.

9.- Trullas JC, Cervera C, Benito N, de la Bellacasa JP, Agusti C, Rovira M, et al. Invasive pulmonary aspergillosis in solid organ and bone marrow transplant recipients. Transplant Proc 2005; 37: 4091-3.

10.- Neofytos D, Horn D, Anaissie E, Steinbach W, Olyaei A, Fishman J, et al. Epidemiology and outcome of invasive fungal infection in adult hematopoietic stem cell transplant recipients: analysis of Multicenter Prospective Antifungal Therapy (PATH) Alliance registry. Clin Infect Dis 2009; 48: 265-73.

11.- Pappas P G, Kauffman C A, Andes D, Benjamin D K Jr, Calandra T F, Edwards J E Jr, et al. Clinical practice guidelines for the management of candidiasis: 2009 update by the Infectious Diseases Society of America. Clin Infect Dis 2009; 48: 503-35.

12.- Marr K A, Bow E, Chiller T, Maschmeyer G, Ribaud P, Segal B, et al. Fungal infection prevention after hematopoietic cell transplantation. Bone Marrow Transplant 2009; 44: 483-7.

13.- Paterson D L, Singh N. Invasive aspergillosis in transplant recipients. Medicine 1999; 78: 123-38.

14.- Paya C V. Fungal infections in solid-organ transplantation. Clin Infect Dis 1993; 16: 677-88.

15.- Singh N. Antifungal prophylaxis for solid organ transplant recipients: seeking clarity amidst controversy. Clin Infect Dis 2000; 31: 545-53.

16.- Kanj S S, Welty-Wolf K, Madden J, Tapson V, Baz M A, Davis R D, et al. Fungal infections in lung and heart-lung transplant recipients. Report of 9 cases and review of the literature. Medicine 1996; 75: 142-56.

17.- Pappas P G, Alexander B D, Andes D R, Hadley S, Kauffman C A, Freifeld A, et al. Invasive fungal infections among organ transplant recipients: results of the TransplantAssociated Infection Surveillance Network (TRANSNET). Clin Infect Dis 2010; 50: 1101-11.

18.- Minari A, Husni R, Avery R K, Longworth D L, DeCamp M, Bertin M, et al. The incidence of invasive aspergillosis among solid organ transplant recipients and implications for prophylaxis in lung transplants. Transpl Infect Dis 2002; 4: 195-200.

19.- Singh N. Antifungal prophylaxis in solid-organ transplant recipients: considerations for clinical trial design. Clin Infect Dis 2004; 39 Suppl 4: S200-6.

20.- Montoya J G, Giraldo L F, Efron B, Stinson E B, Gamberg P, Hunt S, et al. Infectious complications among 620 consecutive heart transplant patients at Stanford University Medical Center. Clin Infect Dis 2001; 33: 629-40.

21.- Hofflin J M, Potasman I, Baldwin J C, Oyer P E, Stinson E B, Remington J S. Infectious complications in heart transplant recipients receiving cyclosporine and corticosteroids. Ann Intern Med 1987; 106 : 209-16.

22.- Hummel M, Thalmann U, Jautzke G, Staib F, Seibold M, Hetzer R. Fungal infections following heart transplantation. Mycoses 1992; 35: 23-34.

23.- Grossi P, Farina C, Fiocchi R, Dalla Gasperina D. Prevalence and outcome of invasive fungal infections in 1,963 thoracic organ transplant recipients: a multicenter retrospective study. Italian Study Group of Fungal Infections in Thoracic Organ Transplant Recipients. Transplantation 2000; 70: 112-6.

24.- Wu G, Vilchez R A, Eidelman B, Fung J, Kormos R, Kusne S. Cryptococcal meningitis: an analysis among 5,521 consecutive organ transplant recipients. Transpl Infect Dis 2002; 4: 183-8.

25.- Davis J A, Horn D L, Marr K A, Fishman J A. Central nervous system involvement in cryptococcal infection in individuals after solid organ transplantation or with AIDS. Transpl Infect Dis 2009; 11: 432-7.

26.- Singh N, Husain S. Invasive aspergillosis in solid organ transplant recipients. Am J Transplant 2009; 9 Suppl 4: S180-91.

27.- Tomblyn M, Chiller T, Einsele H, Gress R, Sepkowitz K, Storek J, et al. Guidelines for preventing infectious complications among hematopoietic cell transplantation recipients: a global perspective. Biol Blood Marrow Transplant 2009; 15: 1143-238.

28.- Pagano L, Caira M, Nosari A, Van Lint M T, Candoni A, Offidani M, et al. Fungal infections in recipients of hematopoietic stem cell transplants: results of the SEIFEM B-2004 study-Sorveglianza Epidemiologica Infezioni Fungine Nelle Emopatie Maligne. Clin Infect Dis 2007; 45: 1161-70.

29.- Kontoyiannis D P, Marr K A, Park B J, Alexander B D, Anaissie E J, Walsh T J, et al. Prospective surveillance for invasive fungal infections in hematopoietic stem cell transplant recipients, 2001-2006: Overview of the Transplant-Associated Infection Surveillance Network (TRANSNET) Database. Clin Infect Dis 2010; 50: 1091-100.

30.- Post M J, Lass-Floerl C, Gastl G, Nachbaur D. Invasive fungal infections in allogeneic and autologous stem cell transplant recipients: a single-center study of 166 transplanted patients. Transpl Infect Dis 2007; 9: 189-95.

31.- Marr K A, Carter R A, Boeckh M, Martin P, Corey L. Invasive aspergillosis in allogeneic stem cell transplant recipients: changes in epidemiology and risk factors. Blood 2002; 100: 4358-66.

32.- Rovira M, Camps I R. Infections in stem cell transplantation. Enf Infec Microbiol Clin 2007; 25: 477-86

33.- Segal B H. Aspergillosis. New Engl J Med 2009; 360: 1870-84.

34.- Hadley S, Huckabee C, Pappas P G, Daly J, Rabkin J, Kauffman C A, et al. Outcomes of antifungal prophylaxis in high-risk liver transplant recipients. Transpl Infect Dis 2009; 11: 40-8

35.- Fortun J, Martin-Davila P, Moreno S, De Vicente E, Nuno J, Candelas A, et al. Risk factors for invasive aspergillosis in liver transplant recipients. Liver Transpl 2002; 8: 1065-70

36.- Verma A, Wade J J, Cheeseman P, Samaroo B, Rela M, Heaton N D, et al. Risk factors for fungal infection in paediatric liver transplant recipients. Pediatr Transplant 2005; 9: $220-5$

37.- Singh N, Aguado J M, Bonatti H, Forrest G, Gupta K L, Safdar N, et al. Zygomycosis in solid organ transplant recipients: a prospective, matched case-control study to assess risks for disease and outcome. J Infect Dis 2009; 200: 1002-11.

38.- Muñoz P, Rodríguez C, Bouza E, Palomo J, Yañez J F, Domínguez M J, et al. Risk factors of invasive aspergillosis after heart transplantation: protective role of oral itraconazole prophylaxis. Am J Transplant 2004; 4: 636-43.

39.- Raviv Y, Kramer M R, Amital A, Rubinovitch B, Bishara J, Shitrit D. Outbreak of aspergillosis infections among lung transplant recipients. Transpl Int 2007;20: 135-40.

40.- Tomblyn M, Chiller T, Einsele H, Gress R, Sepkowitz K, Storek J, et al. Guidelines for preventing infectious complications among hematopoietic cell transplant recipients: a global perspective. Preface. Bone Marrow Transplant 2009; 44: 453-5.

41.- Barnes P D, Marr K A. Risks, diagnosis and outcomes of invasive fungal infections in haematopoietic stem cell transplant recipients. Br J Haematol 2007; 139: 519-31.

42.- Berthelot P, Loulergue P, Raberin H, Turco M, Mounier C, Tran Manh Sung R, et al. Efficacy of environmental measures to decrease the risk 
of hospital-acquired aspergillosis in patients hospitalised in haematology wards. Clin Microbiol Infect 2006; 12: 738-44.

43.- García-Vidal C, Upton A, Kirby K A, Marr K A. Epidemiology of invasive mold infections in allogeneic stem cell transplant recipients: biological risk factors for infection according to time after transplantation. Clin Infect Dis 2008; 47: 1041-50.

44.- Panackal A A, Li H, Kontoyiannis D P, Mori M, Perego C A, Boeckh M, et al. Geoclimatic influences on invasive aspergillosis after hematopoietic stem cell transplantation. Clin Infect Dis 2010; 50: 1588-97.

45.- Kontoyiannis D P, Chamilos G, Lewis R E, Giralt S, Cortés J, Raad I I, et al. Increased bone marrow iron stores is an independent risk factor for invasive aspergillosis in patients with high-risk hematologic malignancies and recipients of allogeneic hematopoietic stem cell transplantation. Cancer 2007; 110: 1303-6.

46.- Bochud P Y, Calandra T. A new step toward individualized antifungal prevention in hematopoietic stem cell transplantation. Clin Infect Dis 2009; 49: 733-5.

47.- Hellinger W C, Bonatti H, Yao J D, Álvarez S, Brumble L M, Keating M R, et al. Risk stratification and targeted antifungal prophylaxis for prevention of aspergillosis and other invasive mold infections after liver transplantation. Liver Transpl 2005; 11: 656-62.

48.- Winston D J, Pakrasi A, Busuttil R W. Prophylactic fluconazole in liver transplant recipients. A randomized, double-blind, placebo-controlled trial. Ann Intern Med 1999; 131: 729-37.

49.- Playford E G, Webster A C, Sorell T C, Craig J C. Antifungal agent for preventing fungal infections in solid organ transplant recipients. Cochrane Database Syst Rev 2004; CD004291.

50.- Cruciani M, Mengoli C, Malena M, Bosco O, Serpelloni G, Grossi P. Antifungal prophylaxis in liver transplant patients: a systematic review and meta-analysis. Liver Transpl 2006; 12: 850-8.

51.- Eschenauer G A, Lam S W, Carver P L. Antifungal prophylaxis in liver transplant recipients. Liver Transpl 2009; 15: 842-58.

52.- Shah T, Lai W K, Gow P, Leeming J, Mutimer D. Low-dose amphotericin for prevention of serious fungal infection following liver transplantation. Transpl Infect Dis 2005;

\section{7: 126-32}

53.- Singh N, Paterson D L, Gayowski T, Wagener M M, Marino I R. Preemptive prophylaxis with a lipid preparation of amphotericin B for invasive fungal infections in liver transplant recipients requiring renal replacement therapy. Transplantation 2001; 71: 910-3.

54.- Fortun J, Martin-Davila P, Montejo M, Muñoz P, Cisneros J M, Ramos A, et al. Prophylaxis with caspofungin for invasive fungal infections in high-risk liver transplant recipients. Transplantation 2009; 87: 424-35.

55.- Husain S, Paterson D L, Studer S, Pilewski J, Crespo M, Zaldonis D, et al. Voriconazole prophylaxis in lung transplant recipients. Am J Transplant 2006; 6: 3008-16.

56.- Gavalda J, Román A. Infection in lung transplantation. Enferm Infecc Microbiol Clin 2007; 25: 639-49; quiz 1650.

57.- Goodman J L, Winston D J, Greenfield R A, Chandrasekar P H, Fox B, Kaizer H, et al. A controlled trial of fluconazole to prevent fungal infections in patients undergoing bone marrow transplantation. New Engl J Med 1992; 326: 845-51.

58.- Marr K A, Seidel K, Slavin M A, Bowden R A, Schoch H G, Flowers M E, et al. Prolonged fluconazole prophylaxis is associated with persistent protection against candidiasisrelated death in allogeneic marrow transplant recipients: long-term follow-up of a randomized, placebo-controlled trial. Blood 2000; 96: 2055-61.

59.- Marr K A, Seidel K, White T C, Bowden R A. Candidemia in allogeneic blood and marrow transplant recipients: evolution of risk factors after the adoption of prophylactic fluconazole. J Infect Dis 2000; 181: 309-16.

60.- Nucci M, Biasoli I, Akiti T, Silveira F, Solza C, Barreiros G, et al. A double-blind, randomized, placebo-controlled trial of itraconazole capsules as antifungal prophylaxis for neutropenic patients. Clin Infect Dis 2000; 30: 300-5.

61.- Winston D J, Maziarz R T, Chandrasekar P H, Lazarus H M, Goldman M, Blumer J L, et al. Intravenous and oral itraconazole versus intravenous and oral fluconazole for long-term antifungal prophylaxis in allogeneic hematopoietic stem-cell transplant recipients. A multicenter, randomized trial. Ann Intern Med 2003; 138: 705-13.
62.- Marr K A, Crippa F, Leisenring W, Hoyle M, Boeckh M, Balajee S A, et al. Itraconazole versus fluconazole for prevention of fungal infections in patients receiving allogeneic stem cell transplants. Blood 2004; 103: 1527-33.

63.- Siwek G T, Pfaller M A, Polgreen P M, Cobb S, Hoth P, Magalheas-Silverman M, et al. Incidence of invasive aspergillosis among allogeneic hematopoietic stem cell transplant patients receiving voriconazole prophylaxis. Diag Microbiol Infect Dis 2006; 55: 209-12.

64.- Ullmann A J, Lipton J H, Vesole D H, Chandrasekar P, Langston A, Tarantolo S R, et al. Posaconazole or fluconazole for prophylaxis in severe graft-versus-host disease. New Engl J Med 2007; 356: 335-47.

65.- Wolff S N, Fay J, Stevens D, Herzig R H, Pohlman B, Bolwell B, et al. Fluconazole vs low-dose amphotericin $B$ for the prevention of fungal infections in patients undergoing bone marrow transplantation: a study of the North American Marrow Transplant Group. Bone Marrow Transplant 2000; 25: 853-9.

66.- Penack O, Schwartz S, Martus P, Reinwald M, Schmidt-Hieber M, Thiel E, et al. Low-dose liposomal amphotericin $\mathrm{B}$ in the prevention of invasive fungal infections in patients with prolonged neutropenia: results from a randomized, single-center trial. Ann Oncol 2006; 17: 1306-12.

67.- Rijnders B J, Cornelissen J J, Slobbe L, Becker M J, Doorduijn J K, Hop W C, et al. Aerosolized liposomal amphotericin B for the prevention of invasive pulmonary aspergillosis during prolonged neutropenia: a randomized, placebo-controlled trial. Clin Infect Dis 2008; 46: 1401-8.

68.- van Burik J A, Ratanatharathorn V, Stepan D E, Miller C B, Lipton J H, Vesole D H, et al. Micafungin versus fluconazole for prophylaxis against invasive fungal infections during neutropenia in patients undergoing hematopoietic stem cell transplantation. Clin Infect Dis 2004; 39: 1407-16.

69.- Cornely O A, Bohme A, Buchheidt D, Einsele H, Heinz W J, Karthaus M, et al. Primary prophylaxis of invasive fungal infections in patients with hematologic malignancies. Recommendations of the Infectious Diseases Working Party of the German Society for Haematology and Oncology. Haematologica 2009; 94: 113-22. 